\title{
Velocity Contour Weighting Method. II: Evaluation in Trapezoidal Channels and Roughness Sensitivity
}

\author{
Daniel J. Howes
}

\author{
and Brett F. Sanders
}

\begin{abstract}
The Velocity Contour Weighting Method (VCWM) was developed in Part I to accurately estimate the cross-sectional average velocity of a prismatic channel flow using acoustic Doppler velocity meter (ADVM) measurements of centerline velocity. Here, the VCWM is validated by its successful application to 25 different concrete-lined trapezoidal channels used for irrigation water delivery. At each site, the cross-sectional distribution of velocity is measured by an acoustic Doppler velocimeter (ADV), which is moved horizontally and vertically through a sampling grid. Multiple tests at some sites led to a total of 51 sets of cross-sectional measurements. ADVM measurements are simulated by interpolating ADV measurements along a set of vertically aligned centerline coordinates typical of ADVM deployments. Subsequent application of the VCWM gives an estimate of the cross-sectional average velocity. Secondly, the velocity-area method is applied to the ADV data to directly measure the cross-sectional average velocity for comparison with the VCWM estimate. Based on this comparison, relative percent errors in the VCWM for all 51 tests were within $\pm 6.3 \%$ using a probable surface roughness $\left(k_{s}\right)$ for the finished concrete of $0.0006 \mathrm{~m}$ without calibration. A sensitivity analysis shows that a range of realistic roughness values for finished concrete can be used without degrading the accuracy of the cross-sectional average velocity predictions by more than an additional $1 \%$. Hence, the method is relatively insensitive to poorly characterized roughness values.
\end{abstract}

\section{Introduction}

The Velocity Contour Weighting Method (VCWM) is designed as a cost-effective methodology for flow measurement in prismatic, lined channels. VCWM requires that the acoustic Doppler velocity meter (ADVM) sensor be installed in an upward-looking configuration along the channel centerline with cabling to the channel bank, where an access box is constructed to handle power, communications, and control hardware (Styles et al. 2006). The simplicity of the ADVM means that many existing channels can easily be retrofitted at low cost to support flow measurement with no head-loss requirement. The most common method of estimating channel-flow measurement today with ADVM technology is the velocity-index rating method (Morlock et al. 2002). However, the velocity-indexing method can be time-consuming and costly to implement because of its calibration requirements. Comparisons between the ADVM sample velocity and cross-sectional average velocity are needed for at least 10 individual flow and depth conditions (Styles et al. 2006).

VCWM is predicated on the nonlinear weighting of streamwise velocity measurements taken over the depth of the centerline water column. The weights represent dimensionless cross sections associated with each velocity measurement, and in Part I an equation was devised to predict the weights as a function of the location of the maximum velocity region $\left(z_{U \max }\right)$ and distance between sampling points $(\Delta z)$. The weights automatically adjust to the velocity distribution because the contour values separating cross sections are based on velocity measurements.

VCWM assumes that velocity readings near the channel bottom will not be sampled by the ADVM, because of a device blanking distance (distance from sensor to first measurement) combined with the device and mount height if installed on the channel bottom. The term "buffer distance" will be used to indicate the total vertical distance from the channel bottom to the first velocity measurement, which includes blanking distance and ADVM height. To estimate the average velocity in this region, a power law is fit to the monotonically increasing ADVM-measured velocities, and the average velocity is estimated by integrating the power law. The result is a simple equation for the average velocity in the first, near-bed area, which in Part I was shown to depend on the channel geometry, the size of the buffer region, and the channel bed roughness $\left(k_{s}\right)$.

The preceding VCWM description shows that all required parameters can readily be measured, except for channel roughness, which must be estimated based on the material properties and finish of the channel. The implication is that VCWM is poised for successful deployment in prismatic, trapezoidal, or rectangular channels without calibration so long as roughness values can be selected based on material properties. Hence, the purpose of this paper is to 
determine the uncertainty of ADVM in field applications and evaluate the range of uncertainty resulting from the use of physically representative roughness parameters.

\section{Methodology}

Field-scale testing is inherently challenging because of logistical constraints, the cost of installing instrumentation, and the lack of control, particularly in comparison to a laboratory setting. Considering this paper's focus on lined prismatic channels, it is very challenging to find a single well-controlled test site, let alone a

Table 1. Number of Tests and Channel Geometry for Each Channel Analyzed

\begin{tabular}{|c|c|c|c|}
\hline $\begin{array}{l}\text { Cross-section } \\
\text { number }\end{array}$ & $\begin{array}{c}\text { Number of } \\
\text { tests }\end{array}$ & $\begin{array}{l}\text { Bottom width } \\
(\mathrm{m})\end{array}$ & $\begin{array}{l}\text { Side slope } \\
\quad(S S)\end{array}$ \\
\hline 1 & 3 & 0.61 & 1.00 \\
\hline 2 & 3 & 0.61 & 0.98 \\
\hline 3 & 5 & 0.61 & 0.98 \\
\hline 4 & 1 & 0.61 & 0.99 \\
\hline 5 & 1 & 0.61 & 1.00 \\
\hline 6 & 1 & 0.61 & 1.00 \\
\hline 7 & 1 & 0.61 & 1.02 \\
\hline 8 & 3 & 0.64 & 1.00 \\
\hline 9 & 3 & 0.61 & 1.00 \\
\hline 10 & 3 & 0.62 & 1.00 \\
\hline 11 & 2 & 0.64 & 1.00 \\
\hline 12 & 2 & 0.61 & 1.00 \\
\hline 13 & 2 & 0.79 & 0.87 \\
\hline 14 & 1 & 0.60 & 1.01 \\
\hline 15 & 2 & 0.61 & 0.99 \\
\hline 16 & 1 & 0.61 & 0.99 \\
\hline 17 & 1 & 0.61 & 1.01 \\
\hline 18 & 1 & 0.61 & 1.00 \\
\hline 19 & 1 & 0.61 & 1.00 \\
\hline 20 & 1 & 0.61 & 1.00 \\
\hline 21 & 2 & 0.30 & 1.00 \\
\hline 22 & 5 & 0.61 & 1.02 \\
\hline 23 & 2 & 0.61 & 1.02 \\
\hline 24 & 3 & 0.61 & 1.00 \\
\hline 25 & 1 & 0.61 & 1.00 \\
\hline
\end{tabular}

set of test sites with varied channel geometries and flow scenarios that would enable the VCWM to be rigorously tested, including an examination of the roughness sensitivity. Note also that the cost of deploying an ADVM is small compared with many other methodologies, but not insignificant. The sensor must be mounted on the channel bottom, preferably in a recessed configuration to minimize the buffer distance. This typically requires that the channel be drained, which is a significant logistical factor.

For this study, an approach was developed based largely on cost and feasibility. A set of 25 different concrete-lined irrigation channels were identified (Table 1). The sites were characterized by prismatic trapezoidal cross sections with a minimum of approximately 10 channel widths of straight unobstructed flow upstream. The channels had bottom widths between 0.3 and $0.79 \mathrm{~m}$ with side slopes $(S S)$ varying between 0.87:1 and 1.02:1 (horizontal:vertical). Water depth varied from $0.39-0.89 \mathrm{~m}$.

At each site, a point velocity measurement device called an acoustic Doppler velocimeter (ADV) was used to measure the velocity in a grid of points that spanned the channel cross section. In many cases, multiple tests were conducted at sites under differing flow rates and depths so that a total of 51 cross-sectional measurements were examined. Fig. 1 shows a typical velocity measurement grid used for the cross-sectional measurements. Semipermanent supports were established so repeat measurements could be taken, and the cross sections were surveyed. The channels were used for water conveyance during the measurement period, so flow rates and water depths for each of the tests were set by system operators, not measurement personnel. However, during the field tests, discharge and water depth were observed to remain constant. Downstream of each measurement site, a control structure maintained the water depth in the channel, resulting in gradually varied flow (GVF) conditions in the measurement reach.

The ADV data were used in two ways to test the VCWM. First, ADV data were used directly by the velocity-area method of discharge estimation (Gupta 1989). This discharge serves as the control. Secondly, using interpolation, the ADV data were used to develop a proxy for a hypothetical centerline ADVM-measured velocity profile. In turn, discharge was estimated by using the VCWM. Both of these methods are described in detail in the following sections.

\section{ADV Measurements}

The cross section of each channel was divided into a grid of vertical and horizontal segments creating a distribution of triangular and rectangular cells. Individual point measurements were taken by

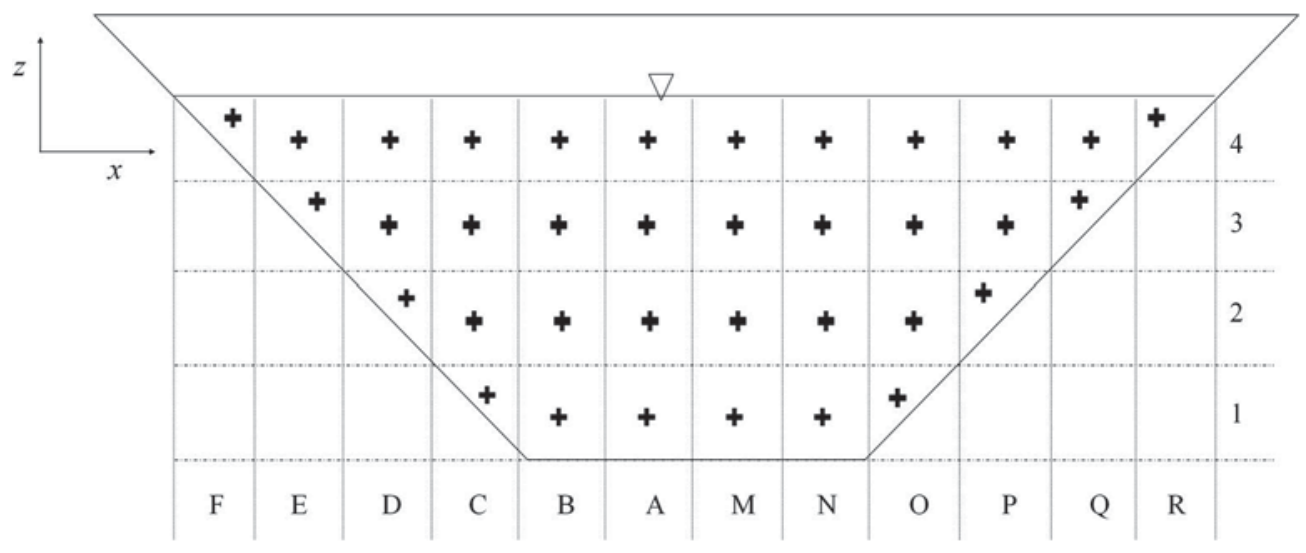

Fig. 1. Example of the cross-sectional segmentation and measured velocity distribution for the tests, where the letters indicate the horizontal location of the measurement, and the numbered values indicate the vertical location 
using an ADV (SonTek/YSI, Inc. Flowtracker) with measurements taken at the centroid of each grid cell (Fig. 1). The actual number of segments and velocity measurements varied based on channel geometry and water depth. The width of each segment was computed based on the channel bottom width so that the horizontal width of each segment was no more than $0.16 \mathrm{~m}$ to minimize the uncertainty in cross-sectional average velocity and the time to take all measurements throughout a cross section because discharge and water level could change if the process was prolonged. For cross sections with larger bottom widths, additional, even numbers of segments were added. The cell heights (vertical dimensions) were determined based on water depth, where the channel was split into a minimum of three vertical sections, and additional sections were added for deeper flows. Cell heights varied between 0.12 and $0.175 \mathrm{~m}$ for the 51 tests.

The flow in each cross-sectional segment was computed by the velocity-area method (Gupta 1989). The observed or control cross-sectional average velocity $(V)$ was computed as the sum of the segment flow divided by the cross-sectional area. Flow depth was measured before and after each set of cross-sectional velocity measurements to ensure constant discharge during the measurement period.

The uncertainty in discharge measurements related the number and size of vertical segments, and the number of velocity measurements was computed by using ISO uncertainty computations based on ISO Standard 748 (SonTek/YSI 2007). The average ISO uncertainty computed using information on measurement technique and ADV for the 51 tests was $3.6 \%$.

An additional issue related to the discharge measurement uncertainty was the location of the measurements in the boundary cells. By measuring the point velocity at the centroid of cells near the channel boundary, the cross-sectional average velocity would be overestimated. The velocity profile near the boundary is nonlinear; therefore, the velocity at the centroid would overestimate the average velocity within these boundary cells. The actual location of the average boundary cell velocity was unknown and would vary depending on cell location either along the side slope or the channel bottom. Because it would be prohibitive in the field to determine the average velocity point in the boundary cells, the measurements were made at the centroid of all cells. By maintaining consistency in the measurement location, a postmeasurement correction was applied to the near-boundary measurements accounting for the lower actual cell average velocity. This procedure was deemed more accurate than trying to estimate exactly where the average velocity point was within the boundary cells.
The postmeasurement correction was developed by using crosssectional velocity data from the computational fluid dynamics model described in Part I. The cross-sectional data from trapezoidal channel scenarios for a roughness equivalent to finished concrete were exported from the model into a Cartesian grid. The segmentation method shown in Fig. 1 was overlaid on the gridded data. Utilizing the CFD velocities within the cells along the boundary, the average cell velocity was computed as the average of the CFD velocities within each cell. The relative percent error between the CFD velocity at the centroid and CFD average cell velocity of each boundary cell is shown in Fig. 2. Interestingly, the centroid CFD velocity was on average $9.1 \%$ greater than the cell average velocity for the triangular cells along the channel side slopes and $6.4 \%$ greater for the rectangular cells along the channel bottom (the standard deviation of the errors was $0.30 \%$ and $0.09 \%$, respectively). The cells adjacent to the triangular cells also contact the channel side slopes and were included in the analysis. As shown in Fig. 2, using the centroid measurement in these adjacent cells led to a mean overestimation of average cell velocity by $1.9 \%$ (with a standard deviation of $0.07 \%$ ).

The postmeasurement correction was applied to the appropriate boundary cells (location dependent, as shown in Fig. 2) for the 51 cross-sectional measurements. Comparing the corrected and uncorrected cross-sectional velocities, it was found that using the uncorrected values would lead to an overestimation in cross-sectional flow of between 1.3 and $3.7 \%$, with higher errors in channels with shallower flows. On average, for the 51 sets of cross-sectional measurements, the postmeasurement correction reduced the uncorrected cross-sectional average velocity by approximately $2.2 \%$.

\section{Interpolation of Vertical Centerline Velocities}

The grid of velocity measurements was used to interpolate the streamwise centerline velocity distribution serving as a proxy for a hypothetical vertical centerline ADVM deployment. As shown in Part I, the VCWM uses centerline velocities at approximately $0.034 \mathrm{~m}$ intervals, from a buffer distance $\left(z_{b}\right)$ to the maximum velocity height $\left(z_{U \max }\right)$, to estimate the cross-sectional average velocity $\left(V_{\mathrm{VCWM}}\right)$. The interpolation of the centerline velocity distribution at $0.034 \mathrm{~m}$ intervals involved two steps. The first was to use natural cubic splines (Kreyszig 2006) to interpolate the centerline velocities at each horizontal (numbered) measurement segment. The second step involved again utilizing natural cubic splines to interpolate between the centerline velocities from the first step to determine the velocities at $0.034 \mathrm{~m}$ intervals vertically along the centerline.

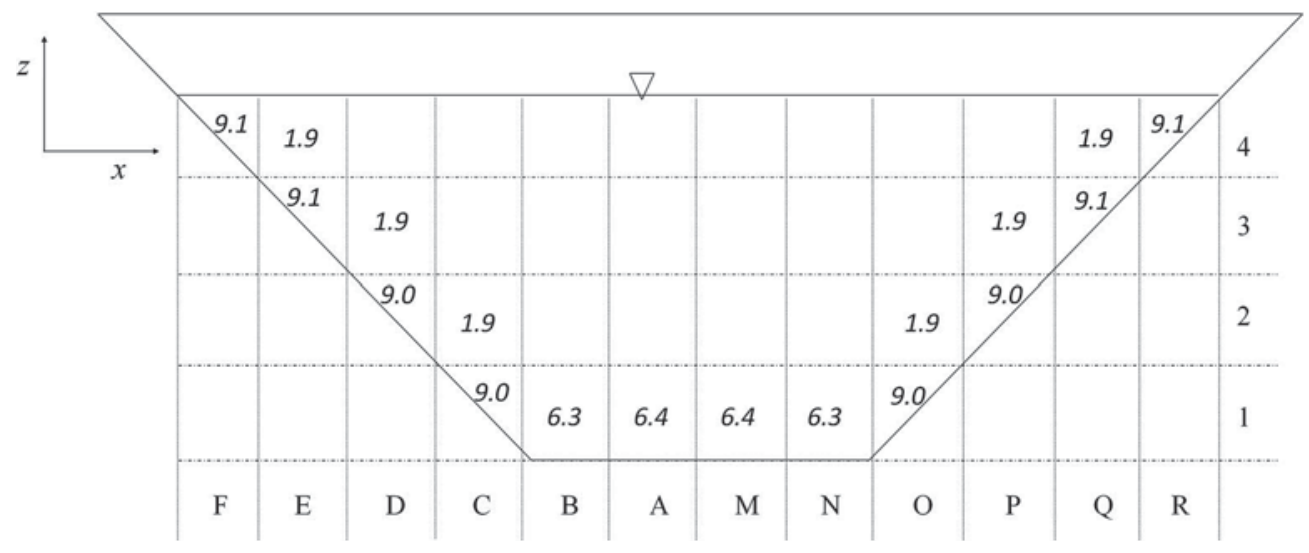

Fig. 2. Example segmentation from Fig. 1 showing relative percent error between the cell centroid velocity and cell average velocity from the CFD analysis 
Because there were even numbers of vertical segments along the channel bottom, the centerline velocities were not directly measured. Measurements were taken approximately $0.08 \mathrm{~m}$ on both sides of the channel's vertical centerline at the centroid of segments A and M. Theoretically, the actual vertical centerline velocity should be greater than the measurements on either side of the centerline, assuming the horizontal velocity profile follows a parabolic shape. Therefore, simply using a linear interpolation between these measurements would underestimate the centerline velocity. To capture the local maxima at the centerline while maintaining the integrity of the measured velocities, cubic splines were chosen. Cubic splines have the lowest interpolation error of all fourth-order interpolation methods (Karpik and Crockett 1997).

These interpolated vertical centerline velocities at each numbered segment were then used to establish the proxy ADVMsampled centerline velocities $\left(U_{i}\right.$, where $\left.2<i<n\right)$ at $0.034 \mathrm{~m}$ intervals from a buffer distance $\left(z_{b}\right)$ of $0.14 \mathrm{~m}$ (corresponding to the first measured velocity, $U_{2}$ ) vertically to $z_{U \max }$ (corresponding to the maximum vertical centerline velocity, $U_{n}$ ). Again, cubic spline interpolation was chosen because of the nonlinearity of the vertical velocity profile. The height of the maximum velocity point $\left(z_{U \max }\right)$ was determined through examination of the interpolated vertical centerline velocities.

\section{Computing $V_{\text {vcwm }}$}

The proxy ADVM centerline velocities $U_{2}$ through $U_{n}$ were used in the VCWM to estimate the cross-sectional average velocity $\left(V_{\mathrm{VCWM}}\right)$, as described in the companion paper. As a reference, the VCWM equations developed in the companion paper are

$$
\begin{gathered}
w_{i}=1.78\left(\frac{\Delta z\left(z_{U \max }-z_{i}\right)}{z_{U \max }^{2}}\right) \\
m=\left(\frac{1.17}{(\mathrm{SS}+0.5)^{0.24}}\right)\left[-2.03 \kappa \sqrt{8} \log \left(\frac{k_{s}}{12.2 R_{h}}\right)\right] \\
C_{a}=1.65\left(\frac{z_{b}}{z_{b \max }}\right)+0.9 \\
\frac{U_{1}}{V_{\mathrm{ADVM}}}=\left[\frac{\left(C_{a} m+1\right) z_{b}^{1 / m}}{C_{a} h^{1 / m}(m+1)}\right] \\
V_{\mathrm{VCWM}}=\sum_{i=2}^{n} w_{i} U_{i}+\left(1-\sum_{i=2}^{n} w_{i}\right) U_{1}
\end{gathered}
$$

where $0.5(h)<z_{U \max }<h$; and $4<m<12$. The channel bottom width, side slope $(S S)$, and water depth $(h)$ measurements were used to compute hydraulic radius $\left(R_{h}\right)$. The weights $\left(w_{i}\right)$ were computed on the basis of these parameters along with $z_{U \max }$, which was estimated as previously described. The average velocity in the buffer region $\left(U_{1}\right)$ was computed for each set of measurements based on the $z_{b}$ of $0.14 \mathrm{~m}, R_{h}, \mathrm{SS}, h$, and surface roughness $\left(k_{s}\right)$.

The uniform concrete-lined channels analyzed were constructed by using a slip form, which provides uniformity and a smooth finish. Values of surface roughness can vary for different levels of concrete finish and aging. Roughness values provided by Henderson (1966) show a range of potential values for smoothed finished formed concrete channels between 0.0003 and $0.0015 \mathrm{~m}$. Concrete channels with very smooth cement-plastered surfaces can be found at the lower $k_{s}$ of $0.0003 \mathrm{~m}$. The $k_{s}$ increases to $0.0006 \mathrm{~m}$ for a smooth uniform channel cast against steel forms to a $k_{s}$ of $0.0015 \mathrm{~m}$ for smoothed finished gunite or shot concrete. Through visual inspection, it was determined that all channels in this study fit into the category related to a $k_{s}$ of $0.0006 \mathrm{~m}$.

\section{Surface Roughness Sensitivity}

A sensitivity analysis was conducted on the $k_{s}$ parameter because roughness is an estimated value that is difficult to directly measure. Unlike the published table of $k_{s}$ values in Henderson (1966), which provides detailed descriptions of the surface related to specific $k_{s}$ values, some published tables in hydraulic textbooks give a range of $k_{s}$ values for channel boundary layer material without describing the finish in detail. Some commonly used references provide $k_{s}$ ranges for finished concrete between 0.0003 and $0.003 \mathrm{~m}$ (Chow 1959; U.S. Bureau of Reclamation 1997; Chanson 2004).

The sensitivity analysis involved applying a lower, middle, and upper $k_{s}$ value to the VCWM from the range provided for finished concrete channels and to examine the effect of $k_{s}$ on the prediction of cross-sectional average velocity. The middle value selected for $k_{s}$ was $0.0015 \mathrm{~m}$, the low value was selected to be $0.0003 \mathrm{~m}$, and the high value analyzed for the sensitivity analysis was $0.003 \mathrm{~m}$. A total of four $k_{s}$ values were examined, including the $0.0006 \mathrm{~m}$ value used for the validation portion of the analysis.

The relative error (percent) between $V_{\mathrm{VCWM}}$ and $V$ was computed using Eq. (1):

$$
\text { Relative error }=\frac{\left(V_{\mathrm{VCWM}}-V\right)}{V} \times 100
$$

\section{Results and Discussion}

The results of the VCWM validation analysis with $k_{s}=0.0006 \mathrm{~m}$ for the 51 sets of cross-sectional measurements are shown in Fig. 3. The corrected observed or control cross-sectional velocity $(V)$ computed from ADV data, using the velocity-area method, are compared with the predicted cross-sectional velocity computed using VCWM $\left(V_{\mathrm{VCWM}}\right)$. As references, lines for a 1-to-1 relationship, $\pm 5 \%$ relative error, and $\pm 10 \%$ relative error are also shown on Fig. 3. The mean relative percent error for all 51 measurements was $-0.37 \%$ with the minimum and maximum relative error of $-4.36 \%$ and $+6.26 \%$, respectively.

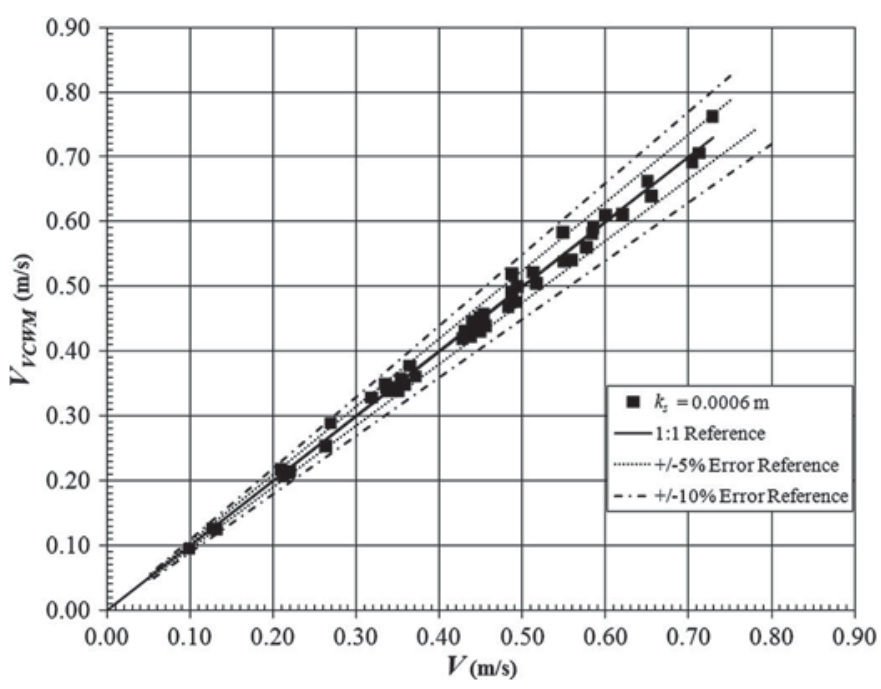

Fig. 3. Comparison of observed and predicted cross-sectional average velocity using VCWM for validation of the method in typical uniform channel cross section consisting of finished concrete with an estimated $k_{s}=0.0006 \mathrm{~m}$ for all channels 

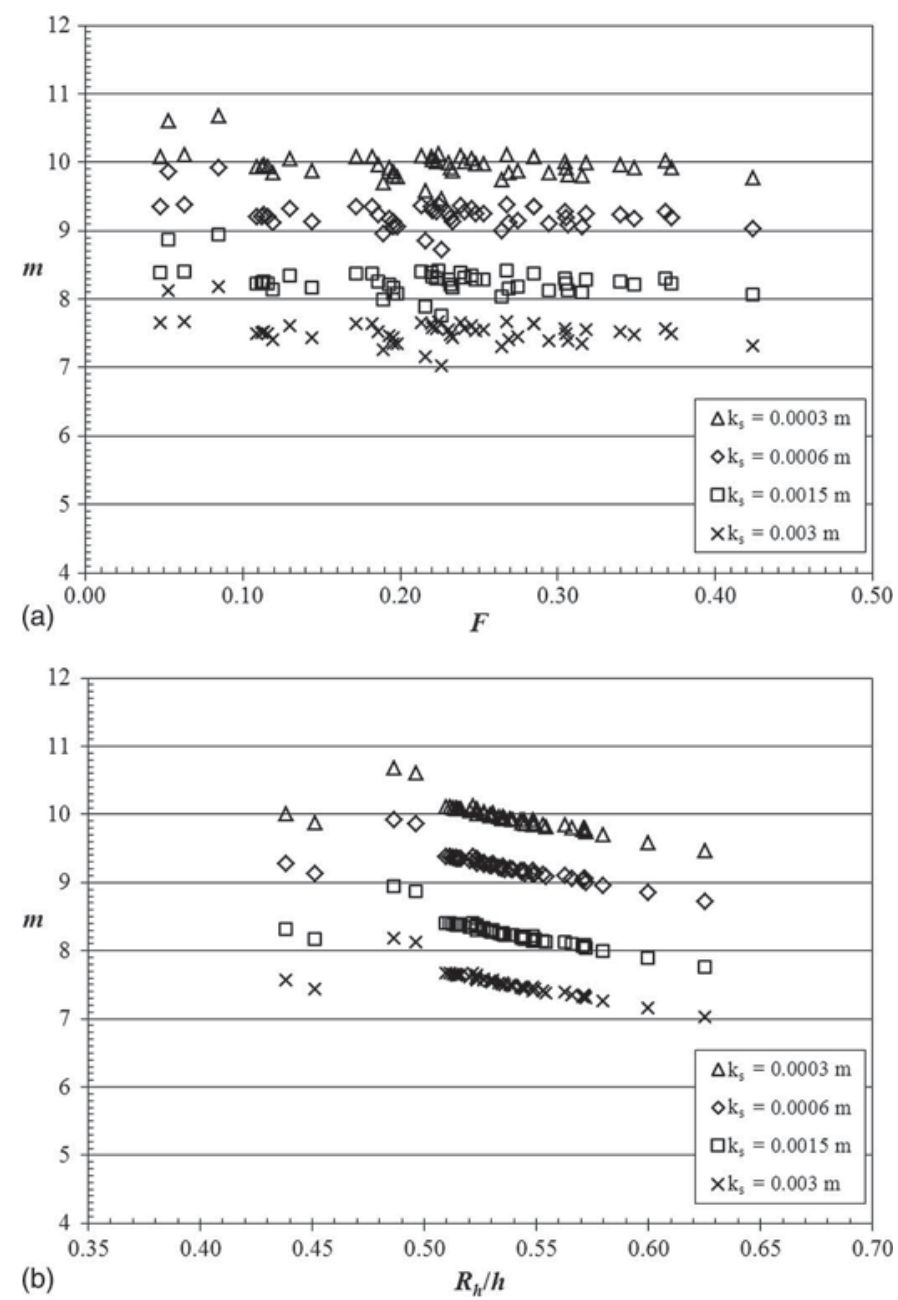

Fig. 4. Variation in the power-law exponent used to estimate $U_{1}$ for the four $k_{s}$ values tested compared with the (a) Froude number; (b) dimensionless hydraulic radius

The indeterminate $k_{s}$ value is a parameter in the computation of the power-law exponent, $1 / m$ for the computation of the buffer region velocity $\left(U_{1}\right)$ component of VCWM. Fig. 4 shows the variation in $m$ for each of the $k_{s}$ values examined in the sensitivity analysis plotted against the Froude number $(F)$ and a dimensionless hydraulic radius. The values of $m$ show significant variability ranging from 6.8-10.7 depending on the roughness and geometry investigated but appear to be insensitive to $F$.

$V_{\text {VCWM }}$ is compared to $V$ for the two bookend surface roughness values, $k_{s}=0.0003 \mathrm{~m}$ and $k_{s}=0.003 \mathrm{~m}$, shown in Fig. 5(a) and 5(b), respectively, and in Table 2. As expected, the higher $k_{s}$ value results in a lower $V_{\mathrm{VCWM}}$ when compared with the lower $k_{s}$ value. However, the significant variation in $m$ at different roughnesses does not translate into significant error within the $V_{\mathrm{VCWM}}$ computation. The tenfold increase in $k_{s}$ only resulted in a difference in mean relative error of approximately $2.74 \%(0.32 \%$ to $-2.42 \%$ from Table 2). This range of relative error was consistent over all 51 cross-sectional measurements investigated.

In all likelihood, the roughness selected would be closer to the actual value than the tenfold difference examined for the sensitivity analysis. The large $k_{s}$ value of $0.003 \mathrm{~m}$ is well outside the range that would logically be selected for the finished concrete-lined cross sections examined in this study. The realistic range for finished concrete sections is $0.0003 \mathrm{~m}$ to $0.0015 \mathrm{~m}$. An examination of
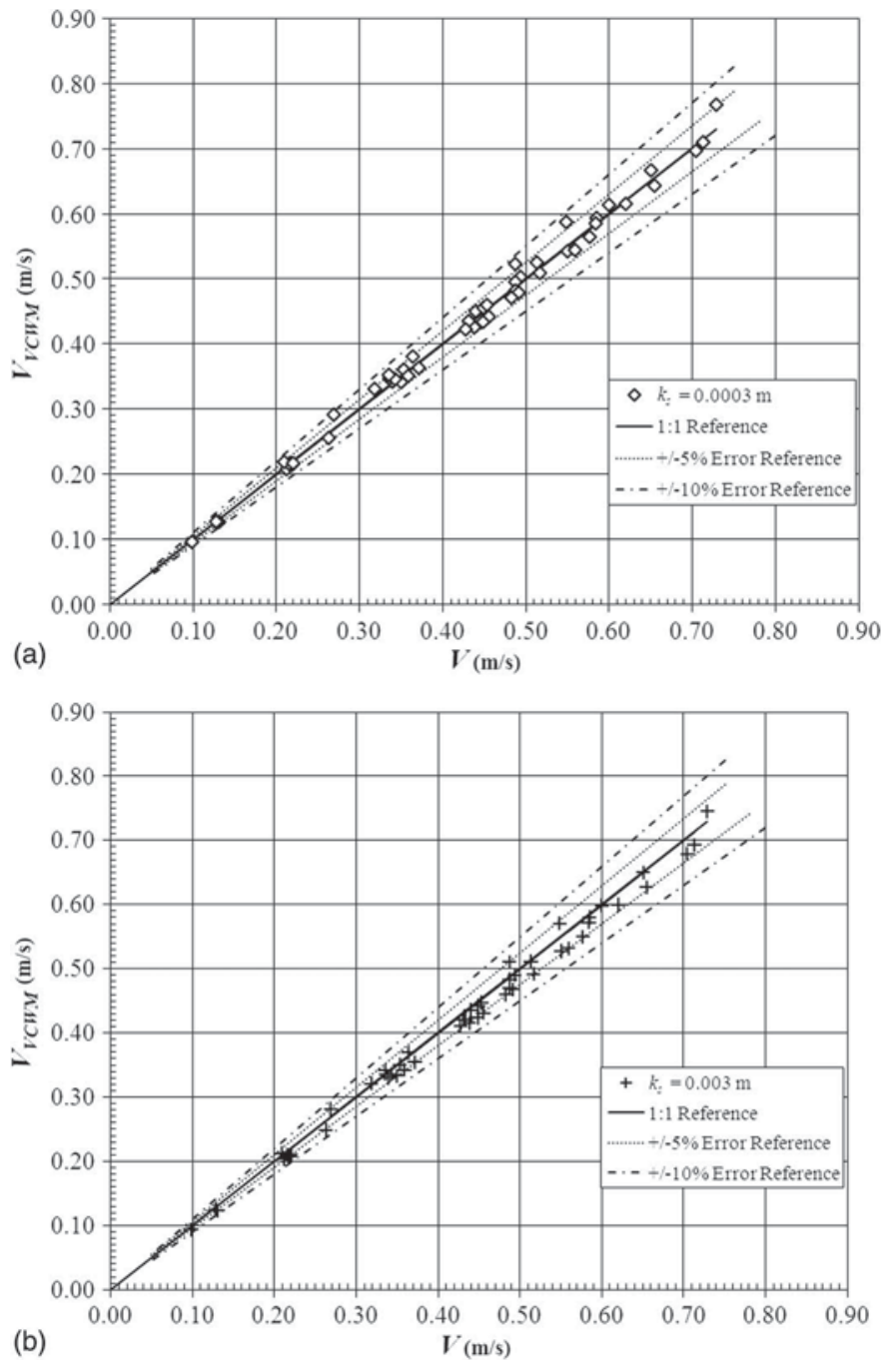

Fig. 5. Computed cross-sectional average velocity using the VCWM $\left(V_{\mathrm{VCWM}}\right)$ compared with the observed cross-sectional average velocity $(V)$ at the edge of the roughness range for finished concrete: (a) $k_{s}=0.0003 \mathrm{~m}$; (b) $k_{s}=0.003 \mathrm{~m}$

the mean relative errors in Table 2 for $k_{s}$ values from 0.0003 $0.0015 \mathrm{~m}$ shows the range of uncertainty related to the $k_{s}$ parameter is $0.32 \%$ and $-1.44 \%$, respectively (approximately a $1.76 \%$ difference). By selecting a $k_{s}$ value in the middle of this range, such as $0.0006 \mathrm{~m}$, the uncertainty can be evaluated by examining the mean relative errors from Table 2 for $k_{s}$ values of 0.0003 and $0.0006 \mathrm{~m}$ (the difference between $0.32 \%$ and $-0.37 \%$ is $0.69 \%$ ) and for $k_{s}$ values of 0.0006 and $0.0015 \mathrm{~m}$ (the difference between $-0.37 \%$ and $-1.44 \%$ is $1.07 \%$ ). Therefore, the likely uncertainty related

Table 2. Mean, Median, and Absolute Minimum and Maximum Relative Errors for the Sensitivity Analysis Examining Four Surface Roughness Values on the 51 Cross-Sectional Measurements

\begin{tabular}{lrrrr}
\hline & \multicolumn{4}{c}{ Surface roughness, $k_{s}$} \\
\cline { 2 - 5 } Relative error & $0.0003 \mathrm{~m}$ & $0.0006 \mathrm{~m}$ & $0.0015 \mathrm{~m}$ & $0.003 \mathrm{~m}$ \\
\hline Mean & $0.32 \%$ & $-0.37 \%$ & $-1.44 \%$ & $-2.42 \%$ \\
Median & $-0.42 \%$ & $-1.05 \%$ & $-2.04 \%$ & $-2.88 \%$ \\
Absolute minimum & $-3.66 \%$ & $-4.36 \%$ & $-5.44 \%$ & $-6.42 \%$ \\
Absolute maximum & $7.14 \%$ & $6.26 \%$ & $5.24 \%$ & $4.33 \%$ \\
\hline
\end{tabular}


to selecting a reasonable $k_{s}$ value near the middle of the range of published values for finished concrete would be approximately $\pm 1.0 \%$, assuming the actual $k_{s}$ was between 0.0003 and $0.0015 \mathrm{~m}$.

The VCWM has two independent components, the weighting function and the estimation of buffer region average velocity. Surface roughness is accounted for by the vertical distribution of centerline velocities; consequently, the weighting function has no surface roughness component. The surface roughness estimate is only required for the estimation of buffer region average velocity, which accounts for a portion of the cross-sectional average velocity. The result is a relative insensitivity of VCWM to $k_{s}$, noting that sensitivity is tied to the buffer distance $\left(z_{b}\right)$, increasing with a higher $z_{b}$ and decreasing with a lower $z_{b}$, demonstrating the importance of minimizing the unmeasured region.

It is possible to minimize the buffer region with existing ADVMs by recessing the device below the channel bottom. However, debris and sediment could deposit over the sensors, preventing them from functioning properly. An investigation is underway utilizing compressed air to remove debris. Initial results are promising; however, the setup increases installation and maintenance costs. As sensor technology improves in the future, the blanking distance and sensor height could be reduced. However, with a smaller blanking distance, care must be taken so that measurements are taken only outside of any flow-field disturbance caused by an ADVM mounted in the flow path.

\section{Conclusion}

Validation testing in concrete-lined trapezoidal channels showed that VCWM can be used to estimate the cross-sectional average velocity with errors of less than $\pm 6.3 \%$ without calibration. The tests were conducted on 51 cross-sectional velocity distributions under different flow rates, water depths, and channel geometries. The VCWM error is comparable to the $\pm 6 \%$ error using the conventional velocity-index rating method with a recommended 10 calibration points (Styles et al. 2006). For the sake of comparison, the most accurate technology for field installations is the longthroated ramp flume, which can obtain discharge measurements within $\pm 2 \%$ if installed and designed properly (Clemmens et al. 1990). However, traditional flumes, including the long-throated flume, can be cost prohibitive and require significant head loss, which is not always available.

The sensitivity analysis of the surface roughness parameter showed that the accuracy of the VCWM is not impacted by more than an additional $\pm 1 \%$ provided a reasonable roughness value is selected for the channel boundary material. This demonstrates the insensitivity of the VCWM to the surface roughness, assuming the roughness does not change significantly on a seasonal basis. To minimize seasonal and annual changes in surface roughness due to the aquatic weed growth and sedimentation that can occur in lined channels, three to four channels widths upstream and two channel widths downstream of the ADVM should be cleaned regularly (depending on the amount of sedimentation, this may be on a monthly basis).

The concrete-lined channels in this evaluation were relatively small with bottom widths from approximately $0.3-1 \mathrm{~m}$ and side slopes from 0.87-1. Because the VCWM was developed using CFD simulations in relatively small channels (presented in the companion paper) and has been tested in similar situations, there is uncertainty related to how the method will perform under different channel conditions. VCWM testing in larger channels, channels with different boundary material, and channels with more significant side slopes is warranted.
The utility of this method is not necessarily limited to lined channel sections. However, with any flow measurement technique, the dynamic boundary conditions in earthen and natural channel conditions pose significant issues. These issues include sedimentation, erosion, and aquatic weed growth. Even if a method can compute the average cross-sectional velocity accurately, there can be significant uncertainty in discharge computed using the velocityarea method related to the area computation.

\section{Acknowledgments}

The authors would like to acknowledge Ron Bliesner, Past President, and Dr. Andrew Keller, President, Keller-Bliesner Engineering, LLC for providing the cross-sectional velocity distribution data. In addition, we would like to acknowledge Dr. Charles Burt, Chairman, Irrigation Training and Research Center, California Polytechnic State University, San Luis Obispo, for his assistance. The writers appreciate the excellent and constructive comments made by the reviewers, which have strengthened both manuscripts. This research was supported by a grant from the University of California Prosser Trust (PR-020), whose support is gratefully acknowledged.

\section{Notation}

The following symbols are used in this paper:

$A=$ hydraulic or wetted cross-sectional area;

$C_{a}=$ coefficient derived as a function of $z_{b}$;

$\mathrm{F}=$ Froude number;

$h=$ flow depth;

$k_{s}=$ equivalent roughness height;

$m$ = power-law exponent;

$R_{h}=$ hydraulic radius;

$R_{s}=$ channel shape factor;

$S S=$ channel side slope;

$U_{i}=$ velocity measured by the upward-looking ADVM at $z_{i}$;

$U_{\max }=$ maximum velocity along the vertical centerline at $z_{U \max }$

$U_{1}=$ average velocity in the buffer region within $z_{b}^{\prime}$;

$u_{i}=$ velocity at contour boundary;

$V=$ control cross-sectional average velocity computed using velocity-area method;

$V_{\mathrm{ADVM}}=$ depth-averaged velocity from the actual upward-looking ADVM;

$V_{\mathrm{VCWM}}=$ calculated cross-sectional average velocity using VCWM;

$w_{i}=$ weight of velocity $U_{i}$;

$w_{1}=$ weight within buffer region;

$x=$ horizontal distance across the channel;

$z=$ normal distance from channel bottom;

$z_{b}=$ buffer distance determined as the height from the channel bottom to the first ADVM sample;

$z_{b}^{\prime}=$ distance to first velocity contour $\left[z_{b}^{\prime}=z_{b}-0.5(0.034 \mathrm{~m})\right] ;$

$z_{U \max }=$ height at the maximum velocity point;

$\Delta z=$ vertical distance between ADVM velocity samples; and $\kappa=$ von Kármán constant.

\section{References}

Chanson, H. (2004). The hydraulics of open channel flow: An introduction, Elsevier Butterworth-Heinemann, Oxford, UK.

Chow, V. T. (1959). Open-channel hydraulics, McGraw-Hill, New York. 
Clemmens, A. J., Replogle, J. A., and Reinink, Y. (1990). "Field predictability of flume and weir operating conditions." J. Hydraul. Eng., 116(1), 102-118.

Gupta, R. S. (1989). Hydrology and hydraulic systems, Prentice Hall, Englewood Cliffs, NJ.

Henderson, F. M. (1966). Open channel flow, MacMillan, New York.

Karpik, S. R., and Crockett, S. R. (1997). "Semi-Lagrangian algorithm for two-dimensional advection-diffusion equation on curvilinear coordinate meshes." J. Hydraul. Eng., 123(5), 389-401.

Kreyszig, E. (2006). Advanced engineering mathematics, Wiley, New York. Morlock, S. E., Nguyen, H. T., and Ross, J. H. (2002). Feasibility of acoustic Doppler velocity meters for the production of discharge records from U.S. Geological Survey streamflow-gaging stations, USGS, Indianapolis.

SonTek/YSI Inc. (2007). FlowTracker handheld ADV technical manual, San Diego

Styles, S. W., Busch, B., Howes, D., and Cardenas, M. (2006). Nonstandard structure flow measurement evaluation using the flow rate indexing procedure-QIP, Irrigation Training and Research Center, California Polytechnic State Univ., San Luis Obispo, CA.

U.S. Bureau of Reclamation. (1997). Water measurement manual, Dept. of the Interior, Denver. 